\title{
ON THE LIE ALGEBRAS ASSOCIATED WITH PURE MAPPING CLASS GROUPS
}

\author{
R. KAROUI AND V. V. VERSHININ
}

\begin{abstract}
Pure braid groups and pure mapping class groups of a punctured sphere have many features in common. In the paper the graded Lie algebra of the descending central series of the pure mapping class of a sphere is studied. A simple presentation of this Lie algebra is obtained.
\end{abstract}

\section{Contents}

1. Introduction

2. Lie algebra $g r^{*}\left(\mathcal{P} \mathcal{M}_{0, n}\right)$

3. Example

References

\section{INTRODUCTION}

Mapping class group is an important object in Topology, Complex Analysis, Algebraic Geometry and other domains. It is a lucky case when the method of Algebraic Topology works perfectly well, the application of the functor of fundamental group completely solves the topological problem: group of isotopy classes of homeomorphisms is described in terms of automorphisms of the fundamental group of the corresponding surface, as states the Dehn-Nilsen-Baer theorem, see [15], for example.

Let $S_{g, b, n}$ be an oriented surface of the genus $g$ with $b$ boundary components and with a set $Q_{n}$ of $n$ fixed points. Consider the group $\operatorname{Homeo}\left(S_{g, b, n}\right)$ of orientation preserving selfhomeomorphisms of $S_{g, b, n}$ which fix pointwise the boundary (if it exists) and map the set $Q_{n}$ into itself. Orientation reversing homeomorphisms also possible to consider, see [8], for example, but we restrict ourselves to orientation preserving case. Let $\operatorname{Homeo}^{0}\left(S_{g, b, n}\right)$ be the normal subgroup of self-homeomorphisms of $S_{g, b, n}$ which are isotopic to identity. Then the mapping class group $\mathcal{M}_{g, b, n}$ is defined as a factor group

$$
\mathcal{M}_{g, b, n}=\operatorname{Homeo}\left(S_{g, b, n}\right) / \operatorname{Homeo}^{0}\left(S_{g, b, n}\right) .
$$

These groups are connected closely with braid groups. In [19] W. Magnus interpreted the braid group as the mapping class group of a punctured disc with the fixed boundary:

$$
B r_{n} \cong \mathcal{M}_{0,1, n} .
$$

The same way as the braid groups the group $\mathcal{M}_{g, b, n}$ has a natural epimorphism to the symmetric group $\Sigma_{n}$ with the kernel called the pure mapping class group $\mathcal{P} \mathcal{M}_{g, b, n}$, so there exists an exact 
sequence:

$$
1 \rightarrow \mathcal{P} \mathcal{M}_{g, b, n} \rightarrow \mathcal{M}_{g, b, n} \rightarrow \Sigma_{n} \rightarrow 1
$$

Geometrically pure mapping class group $\mathcal{P} \mathcal{M}_{g, b, n}$ is descried as consisting of isotopy classes of homeomorphisms that preserve the punctures pointwise.

We call an element $m$ of the pure mapping class group $\mathcal{P} \mathcal{M}_{g, b, n} i$-Makanin or $i$-Brunnian if a homeomorphism $h$ lying in the class $m$

$$
h: S_{g, b, n} \rightarrow S_{g, b, n}
$$

becomes isotopical to the identity map if we fill the deleted point $i$ (or if it becomes non-fixed). Filling the point $i$ generates the homomorphism

$$
p m_{i}: \mathcal{P} \mathcal{M}_{g, b, n} \rightarrow \mathcal{P} \mathcal{M}_{g, b, n-1}
$$

We denote the subgroup of $i$-Makanin elements of the mapping class group by $A_{i}$, it is so the kernel of $p m_{i}$. The subgroups $A_{i}, i=1, \ldots, n$, are conjugate in $\mathcal{M}_{g, b, n}$. The intersection of all subgroups of $i$-Makanin elements is the of Makanin or Brunnian subgroup of the mapping class group

$$
\operatorname{Mak}_{g, b, n}=\cap_{i=1}^{n} A_{i} .
$$

In the paper we consider the pure mapping class group of a sphere with no boundary components $\mathcal{P} \mathcal{M}_{0,0, n}$, which we denote for simplicity by $\mathcal{P} \mathcal{M}_{0, n}$. We study the natural Lie algebra obtained from the descending central series for $\mathcal{P} \mathcal{M}_{0, n}$. One motivation for the work here is that the group $\mathcal{P} \mathcal{M}_{0, n}$ is natural as well as accessible case continuing the same study for the pure braid group done in the works of T. Kohno [17], T. Kohno and T. Oda [18], Y. Ihara [16], R. Bezrukavnikov [4] and for McCool group it was done in the works [7] and [2].

\section{LiE ALGEBRA $g r^{*}\left(\mathcal{P} \mathcal{M}_{0, n}\right)$}

The group $\mathcal{P} \mathcal{M}_{0, n}$ is closely related to the pure braid group on a sphere $P_{n}\left(S^{2}\right)$ as well as its non-pure analogue $\mathcal{M}_{0, n}$ is connected with the (total) braid group of a sphere $B r_{n}\left(S^{2}\right)$.

We start with presentations. Usually the braid group $B r_{n}$ is given by the following Artin presentation [1]. It has the generators $\sigma_{i}, i=1, \ldots, n-1$, and two types of relations:

$$
\begin{cases}\sigma_{i} \sigma_{j} & =\sigma_{j} \sigma_{i}, \quad \text { if }|i-j|>1 \\ \sigma_{i} \sigma_{i+1} \sigma_{i} & =\sigma_{i+1} \sigma_{i} \sigma_{i+1}\end{cases}
$$

The generators $a_{i, j}, 1 \leq i<j \leq n$ for the pure braid group $P_{n}$ (of a disc) can be defined (as elements of the the braid group $B r_{n}$ ) by the formula:

$$
a_{i, j}=\sigma_{j-1} \ldots \sigma_{i+1} \sigma_{i}^{2} \sigma_{i+1}^{-1} \ldots \sigma_{j-1}^{-1} .
$$

Then the defying relations, which are called the Burau relations [5], [22] are as follows:

$$
\left\{\begin{array}{l}
a_{i, j} a_{k, l}=a_{k, l} a_{i, j} \text { for } i<j<k<l \text { and } i<k<l<j, \\
a_{i, j} a_{i, k} a_{j, k}=a_{i, k} a_{j, k} a_{i, j} \text { for } i<j<k \\
a_{i, k} a_{j, k} a_{i, j}=a_{j, k} a_{i, j} a_{i, k} \text { for } i<j<k, \\
a_{i, k} a_{j, k} a_{j, l} a_{j, k}^{-1}=a_{j, k} a_{j, l} a_{j, k}^{-1} a_{i, k} \text { for } i<j<k<l .
\end{array}\right.
$$

It was proved by O. Zariski [26] and then rediscovered by E. Fadell and J. Van Buskirk [9] that a presentation for the braid group of a sphere can be given with the generators $\sigma_{i}, i=1, \ldots, n-1$, 
the same as for the classical braid group, satisfying the braid relations (2.1) and the following sphere relation:

$$
\sigma_{1} \sigma_{2} \ldots \sigma_{n-2} \sigma_{n-1}^{2} \sigma_{n-2} \ldots \sigma_{2} \sigma_{1}=1
$$

Having the same generators, but if we add to the braid relations (2.1) and the sphere relation (2.3) $)$ one more relation ((2.4) below) we get the presentation for the mapping class group of a punctured sphere $\mathcal{M}_{0, n}$ obtained by W. Magnus [19], see also [20] and [21].

$$
\left(\sigma_{1} \sigma_{2} \ldots \sigma_{n-2} \sigma_{n-1}\right)^{n}=1 \text {. }
$$

Let $\Delta$ be the Garside's fundamental word in the braid group $B r_{n}$ [12]. It can be in particular expressed by the formula:

$$
\Delta=\sigma_{1} \ldots \sigma_{n-1} \sigma_{1} \ldots \sigma_{n-2} \ldots \sigma_{1} \sigma_{2} \sigma_{1} .
$$

If we use Garside's notation $\Pi_{t} \equiv \sigma_{1} \ldots \sigma_{t}$, then $\Delta \equiv \Pi_{n-1} \ldots \Pi_{1}$. If the generators $\sigma_{1}, \sigma_{2}, \ldots$, $\sigma_{n-2}, \sigma_{n-1}$, are subject to the braid relations (2.1), then the condition (2.4) is equivalent to the following relation

$$
\Delta^{2}=1
$$

For the pure braid group on a sphere let us introduce the elements $a_{i, j}$ for all $i, j$ by the formulas:

$$
\left\{\begin{array}{l}
a_{j, i}=a_{i, j} \text { for } i<j \leq n, \\
a_{i, i}=1 .
\end{array}\right.
$$

The pure braid group for the sphere has the generators $a_{i, j}$ which satisfy Burau relations (2.2), relations (2.5), and the following relations [13]:

$$
a_{i, i+1} a_{i, i+2} \ldots a_{i, i+n-1}=1 \text { for all } i \leq n,
$$

with the convention that $k+n=k$. Note that $\Delta^{2}$ is a pure braid and it can be expressed by the following formula

$$
\begin{aligned}
& \Delta^{2}=\left(a_{1,2} a_{1,3} \ldots a_{1, n}\right)\left(a_{2,3} a_{2,4} \ldots a_{2, n}\right) \ldots\left(a_{n-1, n}\right)= \\
& \quad\left(a_{1,2}\right)\left(a_{1,3} a_{2,3}\right)\left(a_{1,4} a_{2,4} a_{3,4}\right) \ldots\left(a_{1, n} \ldots \ldots a_{n-1, n}\right) .
\end{aligned}
$$

The fact that this element of the braid group generates its center goes back to Chow [6].

Let us denote by $P_{n}\left(S_{3}^{2}\right)$ the pure braid group on $n$ strings of a sphere with three points deleted or equivalently the subgroup of the pure braid group of a disc on $n+2$ strings where (say, the last) two strings are fixed.

The following statement follows from the normal forms of the groups $P_{n}\left(S^{2}\right)$ and $\mathcal{P} \mathcal{M}_{0, n}[13$ ] and on the geometrical level it was expressed in [14]. Note that the groups $P_{2}\left(S^{2}\right)$ and $\mathcal{P} \mathcal{M}_{0,3}$ are trivial.

Theorem 2.1. (i) The pure braid group of a sphere $P_{n}\left(S^{2}\right)$ for $n \geq 3$ is isomorphic to the direct product of the cyclic group of order 2 (generated by $\Delta^{2}$ ) and $\mathcal{P} \mathcal{M}_{0, n}$.

(ii) The pure braid group $P_{n}$ for $n \geq 2$ is isomorphic to the direct product of the infinite cyclic group (generated by $\Delta^{2}$ ) and $\mathcal{P} \mathcal{M}_{0, n+1}$.

(iii) The groups $\mathcal{P} \mathcal{M}_{0, n}$ and $P_{n-3}\left(S_{3}^{2}\right)$ are isomorphic for $n \geq 4$.

The isomorphism of the part (i) of Theorem 2.1 is compatible with the homomorphisms $p_{i}: P_{n}\left(S^{2}\right) \rightarrow P_{n-1}\left(S^{2}\right), p m_{i}: \mathcal{P} \mathcal{M}_{0, n} \rightarrow \mathcal{P} \mathcal{M}_{0, n-1}$ consisting of deleting one string or forgetting one point, so the group of Makanin braids of a sphere coincide with the subgroup of Makanin mapping class of a sphere. 
For a group $G$ the descending central series

$$
G=\Gamma_{1}>\Gamma_{2}>\cdots>\Gamma_{i}>\Gamma_{i+1}>\ldots
$$

is defined by the formulas

$$
\Gamma_{1}=G, \quad \Gamma_{i+1}=\left[\Gamma_{i}, G\right] .
$$

The descending central series of a discrete group $G$ gives rise to the associated graded Lie algebra (over $\mathbb{Z}) g r^{*}(G)$ [24].

$$
g r^{i}(G)=\Gamma_{i} / \Gamma_{i+1} .
$$

A presentation of the Lie algebra $g r^{*}\left(P_{n}\right)$ for the pure braid group can be described as follows [17. It is the quotient of the free Lie algebra $L\left[A_{i, j} \mid 1 \leq i<j \leq n\right]$ generated by elements $A_{i, j}$ with $1 \leq i<j \leq n$ modulo the "infinitesimal braid relations" or "horizontal $4 T$ relations" given by the following three relations:

$$
\left\{\begin{array}{l}
{\left[A_{i, j}, A_{s, t}\right]=0, \text { if }\{i, j\} \cap\{s, t\}=\phi,} \\
{\left[A_{i, j}, A_{i, k}+A_{j, k}\right]=0, \text { if } i<j<k,} \\
{\left[A_{i, k}, A_{i, j}+A_{j, k}\right]=0, \text { if } i<j<k .}
\end{array}\right.
$$

Y. Ihara in [16] gave a presentation of the Lie algebra $g r^{*}\left(P_{n}\left(S^{2}\right)\right)$ of the pure braid group of a sphere. It is convenient to have conventions like (2.5). So, it is the quotient of the free Lie algebra $L\left[B_{i, j} \mid 1 \leq i, j \leq n\right]$ generated by elements $B_{i, j}$ with $1 \leq i, j \leq n$ modulo the following relations:

$$
\left\{\begin{array}{l}
B_{i, j}=B_{j, i} \text { for } 1 \leq i, j \leq n \\
B_{i, i}=0 \text { for } 1 \leq i \leq n \\
{\left[B_{i, j}, B_{s, t}\right]=0, \text { if }\{i, j\} \cap\{s, t\}=\phi} \\
\sum_{j=1}^{n} B_{i, j}=0, \text { for } 1 \leq i \leq n .
\end{array}\right.
$$

It is a factor algebra of the algebra $g r^{*}\left(P_{n}\right)$ : the last two relations in (2) are the consequences of the third and the forth type relations in (2).

Theorem 2.2. (i) The graded Lie algebra $\operatorname{gr}^{*}\left(\mathcal{P} \mathcal{M}_{0, n}\right)$ is the quotient of the free Lie algebra $L\left[B_{i, j} \mid 1 \leq i, j \leq n\right]$ modulo the following relations:

$$
\left\{\begin{array}{l}
B_{i, j}=B_{j, i} \text { for } 1 \leq i, j \leq n \\
B_{i, i}=0 \text { for } 1 \leq i \leq n \\
{\left[B_{i, j}, B_{s, t}\right]=0, \text { if }\{i, j\} \cap\{s, t\}=\phi} \\
\sum_{j=1}^{n} B_{i, j}=0, \text { for } 1 \leq i \leq n, \\
\sum_{i=1}^{n-1} \sum_{j=i+1}^{n} B_{i, j}=0
\end{array}\right.
$$

(ii) The graded Lie algebra $\mathrm{gr}^{*}\left(\mathcal{P} \mathcal{M}_{0, n}\right)$ is the quotient of the free Lie algebra $L\left[A_{i, j} \mid 1 \leq i<\right.$ $j \leq n-1]$ generated by elements $A_{i, j}$ with $1 \leq i<j \leq n-1$ modulo the following relations:

$$
\left\{\begin{array}{l}
{\left[A_{i, j}, A_{s, t}\right]=0, \text { if }\{i, j\} \cap\{s, t\}=\phi,} \\
\sum_{i=1}^{n-2} \sum_{j=i+1}^{n-1} A_{i, j}=0 .
\end{array}\right.
$$


Proof. Part (i) of Theorem 2.6 follows from the Ihara presentation and the part (i) of Theorem 2.1. The pure mapping class group $\mathcal{P} \mathcal{M}_{0, n}$ is a direct summand in the pure braid group of a sphere $P_{n}\left(S^{2}\right)$, so there is no problem in obtaining exact sequence after application of the functor of associated graded Lie algebras $g r^{*}$.

To obtain part (ii) let us write in detail the system of linear equations which constitute the forth part of the Ihara relations (2):

$$
\left\{\begin{array}{l}
B_{1,2}+B_{1,3}+\ldots B_{1, n}=0 \\
B_{1,2}+B_{2,3}+\ldots B_{2, n}=0 \\
\ldots \\
B_{1, n}+B_{2, n}+\ldots B_{n-1, n}=0
\end{array}\right.
$$

The $n-1$ equations (except the last one) give the possibility to exclude the letters $B_{i, n}$ for $1 \leq$ $i \leq n-1$ from the presentation. Note that the third type relations in (2) with $j=n$ are the consequences of the same type relation with $j \leq n-1$ and relations (2.7) except the last one. Take then the linear combination of these equations where the first $n-1$ equations are taken with the coefficient +1 and the last one with the coefficient -1 . We get the equation

$$
2\left(\sum_{i=1}^{n-2} \sum_{j=i+1}^{n-1} A_{i, j}\right)=0 .
$$

The second type relation in (2.6) is a consequence of the last equation in (2) and first relation in (2.7).

Corollary 2.1. A presentation of the Lie algebra $\operatorname{gr}^{*}\left(P_{n}\left(S^{2}\right)\right)$ can be given with generators $A_{i, j}$ with $1 \leq i<j \leq n-1$, modulo the following relations:

$$
\left\{\begin{array}{l}
{\left[A_{i, j}, A_{s, t}\right]=0, \text { if }\{i, j\} \cap\{s, t\}=\phi,} \\
2\left(\sum_{i=1}^{n-2} \sum_{j=i+1}^{n-1} A_{i, j}\right)=0 .
\end{array}\right.
$$

So, the element $\sum_{i=1}^{n-2} \sum_{j=i+1}^{n-1} A_{i, j}$ of order 2 generates the central subalgebra in $g r^{*}\left(P_{n}\left(S^{2}\right)\right)$.

\section{EXAMPLE}

The pure braid group of a sphere $P_{4}\left(S^{2}\right)$ is isomorphic to the direct product of the cyclic group of order 2 (generated by $\Delta^{2}$ ) and the pure braid group on one string of a sphere with three points deleted, that is the fundamental group of disc with two points deleted, that is a free group on two generators $F_{2}$. Its associated graded Lie algebra is a direct sum of central $\mathbb{Z} / 2$ and the free Lie algebra on two generators. The pure mapping class group $\mathcal{P} \mathcal{M}_{0,4}$ is isomorphic to a free group on two generators. According to Theorem 2.2 its associated graded Lie algebra is the quotient of the free Lie algebra $L\left[A_{1,2}, A_{1,3}, A_{2,3}\right]$ modulo the following relation:

$$
A_{1,2}+A_{1,3}+A_{2,3}=0
$$

so, is a free Lie algebra on two generators. 


\section{REFERENCES}

[1] E. Artin, Theorie der Zöpfe. Abh. Math. Semin. Univ. Hamburg, 1925, v. 4, 47-72.

[2] B. Berceanu, S. Papadima, Universal representations of braid and braid-permutation groups, arXiv:0708.0634

[3] J. A. Berrick, F. R. Cohen, Y. L. Wong and J. Wu, Configurations, braids, and homotopy groups. J. Amer. Math. Soc. 19 (2006), no. 2, 265-326.

[4] R. Bezrukavnikov, Koszul DG-algebras arising from configuration spaces. Geom. Funct. Anal. 4 (1994), no. 2, 119-135.

[5] W. Burau, Über Zopfinvarianten. (German) Abh. Math. Semin. Hamb. Univ. 9, 117-124 (1932).

[6] W.-L. Chow, On the algebraical braid group, Ann. Math. 1948, 49, No 3, 654-658.

[7] F. R. Cohen; J. Pakianathan; V. V. Vershinin; J. Wu, Basis-conjugating automorphisms of a free group and associated Lie algebras. Iwase, Norio (ed.) et al., Proc. of the conference on groups, homotopy and configuration spaces, Univ. of Tokyo, July, 2005. Coventry: Geometry and Topology Monographs 13, 147-168 (2008).

[8] W. Dicks and E. Formanek, Algebraic mapping-class groups of orientable surfaces with boundaries. pp. 57-115, in: Infinite groups: geometric, combinatorial and dynamical aspects. Progress in Math. 248, Birkhäuser Verlag, Basel, 2005.

[9] E. Fadell and J. Van Buskirk, The braid groups of $E^{2}$ and $S^{2}$. Duke Math. J. 29 1962, 243-257.

[10] M. Falk, and R. Randell, The lower central series of a fiber-type arrangement, Invent. Math., 82 (1985), 77-88.

[11] B. Farb, C. J. Leininger, D. Margalit The lower central series and pseudo-Anosov dilatations. arXiv:math/0603675.

[12] F. A. Garside, The braid group and other groups, Quart. J. Math. Oxford Ser. 1969, 20, 235-254.

[13] R. Gillette; J. Van Buskirk, The word problem and consequences for the braid groups and mapping class groups of the 2-sphere. Trans. Amer. Math. Soc. 131. 1968. 277-296.

[14] D. L. Gonalves; J. Guaschi, The roots of the full twist for surface braid groups. Math. Proc. Cambridge Philos. Soc. 137 (2004), no. 2, 307-320.

[15] N. V. Ivanov, Mapping class groups, Handbook of geometric topology, 523-633, North-Holland, Amsterdam, 2002.

[16] Y. Ihara, Galois group and some arithmetic functions, Proceedings of the International Congress of mathematicians, Kyoto, 1990, Springer (1991), 99-120.

[17] T. Kohno, Série de Poincaré-Koszul associée aux groupes de tresses pure, Invent. Math., 82 (1985), $57-75$.

[18] T. Kohno; T. Oda, The lower central series of the pure braid group of an algebraic curve. Galois representations and arithmetic algebraic geometry (Kyoto, 1985/Tokyo, 1986), 201-219, Adv. Stud. Pure Math., 12, North-Holland, Amsterdam, 1987.

[19] W. Magnus, Über Automorphismen von Fundamentalgruppen berandeter Flächen. (German) Math. Ann. 109, 617-646 (1934).

[20] W. Magnus, Braid groups: A survey. Proceedings of the Second International Conference on the Theory of Groups (Australian Nat. Univ., Canberra, 1973), pp. 463-487. Lecture Notes in Math., Vol. 372, Springer, Berlin, 1974.

[21] W. Magnus; A. Karrass; D. Solitar, Combinatorial group theory. Presentations of groups in terms of generators and relations. 2nd rev. ed. Dover Books on Advanced Mathematics. New York: Dover Publications, Inc. XII, 444 p. (1976).

[22] A. A. Markoff, Foundations of the Algebraic Theory of Tresses, Trudy Mat. Inst. Steklova, No 16, 1945 (Russian, English summary).

[23] G. P. Scott, Braid groups and the group of homeomorphisms of a surface. Proc. Cambridge Philos. Soc. 68, 1970, 605-617.

[24] J.-P. Serre, Lie algebras and Lie groups. 1964 lectures given at Harvard University. Corrected fifth printing of the second (1992) edition. Lecture Notes in Mathematics, 1500. Springer-Verlag, Berlin, 2006. viii $+168 \mathrm{pp}$.

[25] V. V. Vershinin, Braid groups, their Properties and Generalizations. Handbook of Algebra, vol. 4, Elzseier, Amsterdam a.o. 2006, p. 427-465. 
[26] O. Zariski, On the Poincare group of rational plane curves, Am. J. Math. 1936, 58, 607-619.

Département des Sciences Mathématiques, Université Montpellier II, Place Eugène BatailLON, 34095 Montpellier CedeX 5, France

E-mail address: Rym.Karoui@math.univ-montp2.fr

Département des Sciences Mathématiques, Université Montpellier iI, Place Eugène BatailLON, 34095 MontPellier CEDEX 5, France

E-mail address: vershini@math.univ-montp2.fr

Sobolev Institute of Mathematics, Novosibirsk 630090, Russia

E-mail address: versh@math.nsc.ru 